\title{
Synchrotron Maser From Weakly Magnetised Neutron Stars As The Emission Mechanism Of Fast Radio Bursts
}

\author{
Killian Long* \\ University College Cork, Ireland \\ E-mail: killian.long@umail.ucc.ie \\ Asaf Pe'er \\ Bar Ilan University, Israel \\ E-mail: asaf.peer@biu.ac.il
}

\begin{abstract}
The origin of Fast Radio Bursts (FRBs) is still mysterious. All FRBs to date show extremely high brightness temperatures, requiring a coherent emission mechanism. Using constraints derived from the physics of one of these mechanisms, the synchrotron maser, as well as observations, we show that accretion induced explosions of neutron stars with surface magnetic fields of $B_{*} \lesssim$ $10^{11} \mathrm{G}$ are favoured as FRB progenitors.
\end{abstract}

High Energy Phenomena in Relativistic Outflows VII - HEPRO VII

9-12 July 2019

Facultat de FÃ $\backsim$ sica, Universitat de Barcelona, Spain

\footnotetext{
*Speaker.
} 


\section{Introduction}

Fast Radio Bursts (FRBs) are bright radio transients of millisecond duration. A total of 98 FRBs have been published to date $(1)^{1}$. They have typical fluxes of $\sim 1 \mathrm{Jy}$ and are distinguished by their large dispersion measures (DM). These are in the range $176 \mathrm{pc} \mathrm{cm}^{-3}$ to $2596 \mathrm{pc} \mathrm{cm}^{-3}$, an order of magnitude greater than values expected from Milky Way electrons $(1 ; 2)$, suggesting an extragalactic origin for FRBs.

Of the 98 FRBs to date, 11 have been observed to repeat. The localisation of the first repeater, FRB 121102 (3) allowed a persistent counterpart and host galaxy to be identified at a redshift of $z=0.193$, equivalent to a luminosity distance of $d_{L}=972 \mathrm{Mpc}(4 ; 5 ; 6)$. This, as well as further localisations $(7 ; 8 ; 9)$ provide a strong case for the extragalactic origin of FRBs.

The nature of FRB progenitors is still unknown. The small scale and large energies involved has led most models to consider compact objects such as neutron stars playing crucial roles in the production of FRBs. The numerous proposed models fall into two classes, cataclysmic and non-cataclysmic. Cataclysmic models include binary neutron star mergers (e.g. 10), white dwarf mergers (11) and neutron star-black hole mergers (12). Non-cataclysmic models include giant pulses from extragalactic pulsars and young neutron stars (e.g. 13; 14) and flares from soft gamma repeaters (SGRs) (e.g. 15; 16).

Despite the large degree of uncertainty regarding the nature of the progenitor, there is a consensus on the need for a coherent emission process. This follows from the extremely high brightness temperatures of up to $T_{b} \sim 10^{37} \mathrm{~K}(17)$. As we show here, this requirement holds the key to understanding the nature of the progenitor. Analysing the conditions required to produce the necessary coherent emission allows us to place strong constraints on possible FRB progenitors.

We find that the conditions found in the environments of neutron stars with surface magnetic fields of $B_{*} \lesssim 10^{11} \mathrm{G}$ are similar to those required for a coherent emission mechanism, the synchrotron maser, to produce a FRB. Furthermore, the proportion of neutron stars with these magnetic fields is $\sim 10 \%$, and the FRB rate is comparable to this fraction of the neutron star formation rate. These results allow us to propose weakly magnetised neutron stars as FRB progenitors.

\section{The Basic Physics of the Synchrotron Maser}

Several mechanisms have been proposed to explain the coherent emission required to produce the extreme brightness temperatures of FRBs. Models include coherent curvature emission (18; 19), the cyclotron/synchrotron maser $(15 ; 16 ; 20 ; 21)$ and collisionless Bremsstrahlung in strong plasma turbulence (22).

Here we examine the synchrotron maser as the mechanism responsible for FRBs. The maser has the advantage of being a viable emission mechanism over a range of magnetic fields and number densities, as well as not requiring particles to be bunched in small volumes in order to obtain coherent emission (21). Previous works invoking the maser have examined specific models (15; $16 ; 20$ ) or the mechanism itself (21), but have not used the mechanism's properties to derive general constraints on the progenitor.

\footnotetext{
${ }^{1}$ http://www.frbcat.org/
} 
Maser emission is produced due to interaction between electromagnetic waves and energetic particles in a plasma, which can result in negative absorption and stimulated emission under certain conditions $(23 ; 24)$. The behaviour of the maser is determined by the form of the particle distribution and the environment where it occurs. For masing to occur, a population inversion in the electron distribution is required $(23 ; 25)$.

Here we consider the maser in a weakly magnetised $\left(v_{p} / v_{B}>1\right)$, relativistic, nonthermal plasma, where $v_{p}$ is the plasma frequency, given by $v_{p}=\sqrt{n e^{2} / \pi \gamma m_{e}}$. Here $n$ is the number density of the plasma and $\gamma$ is the Lorentz factor of the electrons. The gyration frequency of the plasma particles, $v_{B}$, is given by $v_{B}=e B /\left(2 \pi \gamma m_{e} c\right)$. Maser emission in these conditions has been proposed as the source of radio emission from gamma ray burst afterglows (26). In this scenario the masing emission is due to the Razin effect, a modification of the emission from a relativistic plasma with respect to the vacuum case, which can result in either suppression or, when a population inversion is present, amplification of the emitted signal $(27 ; 28)$. This is due to a change in the beaming angle of the radiation when the refractive index of the plasma is less than unity (25). For this case, a relativistic plasma is required, as the Razin effect is a relativistic effect and so would not effect cyclotron emission. The emission is strongest near the Razin frequency of $v_{R^{*}} \approx v_{p} \min \left\{\gamma, \sqrt{v_{p} / v_{B}}\right\}$ and is not important in strongly magnetised plasmas, as in this scenario the emission peaks at a frequency below the plasma frequency.

\section{Physical and observational constraints of the allowed parameter space region that enables the production of FRBs}

The physical conditions in the region where the masing takes place can be constrained using the physics of the maser and constraints from observations, allowing us to place limits on the magnetic fields and number densities where the synchrotron maser can plausibly be the emission mechanism for FRBs.

The data enable us to obtain constraints linking the size and number density of the masing region to the magnetic field of the neutron star. These constraints are obtained from: (i) the energetics of the burst and size of the masing region, (ii) the efficiency of the maser mechanism, (iii) the dispersion measure of the burst and (iv) the frequency of the signal.

We consider a cataclysmic FRB progenitor where masing takes place in a spherical shell of thickness $d$, located a distance $R$ from the central object. The maser will be activated by the formation of a population inversion in the shell. The magnetic fields and short timescale $(\lesssim d / c \Gamma)$ required suggest this object is a neutron star, though the timescale for maser emission is given by the duration of the maser itself (21). Assuming a relativistic blast wave the shocked plasma has a width $\sim R / \Gamma(29)$, where $\Gamma$ is the Lorentz factor of the blast wave. This typical width provides the first constraint, on the thickness of the shell: $d \sim R / \Gamma$. The minimum thickness of the shell depends on the number of particles that contribute to the masing, $N_{e}=E /\left(\eta\left\langle E_{e}\right\rangle\right)$, and their number density, $n_{e}$. Here, $E$ is the energy of the bursts (in the range $10^{38}-10^{40} \mathrm{erg}$ for the repeater $(4 ; 30)$ ), $\eta$ is the fraction of the electrons' energy that contributes to the maser and $\left\langle E_{e}\right\rangle$ is the average energy of the masing electrons. These shocked electrons have a thermal energy of $\gamma \approx \Gamma$.

Constraint (ii) originates from the efficiency of the maser. For the maser to be a viable emission mechanism, the growth rate of the signal must be large enough to extract a fraction $\eta$ of the particle 
energy. The maser will be quenched when the maser reaches saturation. The efficiency of the maser mechanism in simulations of relativistic shocks was shown to be $\eta \lesssim 10^{-1}$ (e.g. 31; 32). In the case of AKR, the efficiency is in the range $\eta \sim 10^{-1}-10^{-3}$ (23). The exact value, however, depends on the form of the particle distribution, which is uncertain. We therefore examine values of $\eta$ in the range $10^{-3} \lesssim \eta \lesssim 10^{-1}$ in this work. (33) give upper limits to the efficiency of $\eta \lesssim 10^{-5}$, derived from limits on the brightness temperature from induced Compton scattering. However, plasma experiments suggest that this saturation effect is not observed for high $T_{B}(22 ; 34)$.

The growth rate, and therefore the efficiency, depends on the distribution function of the electrons. There is a wide range of possible distributions which can provide the requisite population inversion. We do not specify an exact form for the distribution as our results are unchanged provided the growth rate is large enough to extract the required energy over the width of the masing cavity.

The third constraint comes from the dispersion measure. Assuming the DM from the source is solely due to the particles in the shell, one has $D M_{\text {source }}=D M_{\text {shell }}$, where $D M_{\text {shell }}=n_{c} d$ for a cold plasma and $D M_{\text {shell }}=n_{e} d / 2 \gamma$ for a relativistic plasma. Here, $n_{c}$ and $n_{e}$ denote the cold and relativistic electrons in the shell (35). The contribution to the DM from the source region is uncertain. The total DM value also contains contributions from the Milky Way, Milky Way halo, the intergalactic medium (IGM) and the host galaxy. For FRB 121102, (4) estimate the DM due to the host galaxy as $55 \lesssim D M_{\text {host }} \lesssim 225 \mathrm{pc} \mathrm{cm}^{-3}$. The contribution to this from the galaxy rather than the source region depends on the location of the FRB within the galaxy. Using these values as guidelines, the DM due to the shell is $D M_{\text {shell }} \lesssim 225 \mathrm{pc} \mathrm{cm}^{-3}$.

Constraint (iv) is derived from equating the masing frequency to the emission frequency of the bursts, which have observed frequencies of approximately $v_{o b s} \sim 1.4 \mathrm{GHz}$. The maser frequency is given by the Razin frequency, $v_{R} *$, where the growth rate is at a maximum. Equating the Razin frequency to the emission frequency of $v_{o b s} / \Gamma$ and noting $\gamma=\Gamma$ gives the magnetic field in the masing region as

$$
B_{M}=1.32 \times 10^{-13} \sqrt{n_{e}^{3} \gamma^{3}} .
$$

The range $1<\frac{v_{p}}{v_{B}}<\gamma^{2}$ delimits the range where $v_{R^{*}}=v_{p} \sqrt{v_{p} / v_{B}}$. For $v_{p} / v_{B}>\gamma^{2}$ the Razin frequency is $v_{R^{*}}=\gamma v_{p}$. While masing emission is still possible in this regime, the allowed parameter space is restricted to a small region with low magnetic fields due to constraints from the DM, shell size and observed emission frequency. Including the neutron stars in this region will not change the statistics for our model discussed below. The range of interest can therefore be expressed in terms of the number density using equation 3.1 as

$$
\frac{2.4 \times 10^{10}}{\gamma^{3}}<n_{e}<\frac{2.4 \times 10^{10}}{\gamma}
$$

This provides upper and lower limits on the number density which depend on the Lorentz factor of the electrons.

\section{Results}

We investigate neutron star surface magnetic fields in the range $10^{7} \mathrm{G}<B_{*}<10^{15} \mathrm{G}$. This 
encompasses the full range of surface magnetic field values for all published pulsars $(36)^{2}$. The magnetic field outside the surface was taken to be of the form $B \propto 1 / r^{3}$ inside the light cylinder, and $B \propto 1 / r$ outside (37). The light cylinder radius $r_{L}=c P / 2 \pi$ is the radius at which the co-rotating speed is equal to the speed of light. Here $P$ is the period of the pulsar. We also investigate the full range of number densities in the masing region.

The range of $n_{e}$ is given by equation 3.2. Lorentz factors of $\gamma=2,5,10,100,10^{3}$ and $10^{6}$ were examined. The larger values were chosen to examine conditions similar to pulsar wind nebulae, which have Lorentz factors of up to $\sim 10^{6}(38 ; 39)$. The allowed parameter space for $\gamma=10$, $E=10^{40} \mathrm{erg}$ and $\eta=10^{-3}$ is shown in Figure 1 as an example. In this case, the results indicate that the allowed parameter space is restricted to low magnetic fields and $n \sim 10^{8} \mathrm{~cm}^{-3}$. Increasing the allowed values of the DM results in the lower limit decreasing.

The allowed surface magnetic field values depend on the Lorentz factor, number density in the masing region and the distance to the masing region, $R$. As the volume of the shell is $V \approx$ $4 \pi R^{2} d$, the distance to the masing region and the number density are related by the expression $R \approx\left(\frac{E}{4 \pi \eta m c^{2} n_{e}}\right)^{1 / 3}$. Using equation 3.1, the surface magnetic field can therefore be expressed as $B_{*} \propto n_{e}^{7 / 6} \gamma^{3 / 2}$. Taking into account the maximum allowed number density from equation 3.2, $n_{e, \max } \propto \gamma^{-1}$, the maximum surface magnetic field is $B_{*, \max } \propto \gamma^{1 / 3}$. Thus, the allowed surface magnetic field depends only weakly on the Lorentz factor. Therefore, even for very large values of $\gamma$ only low values of $B_{*}$ are attainable.

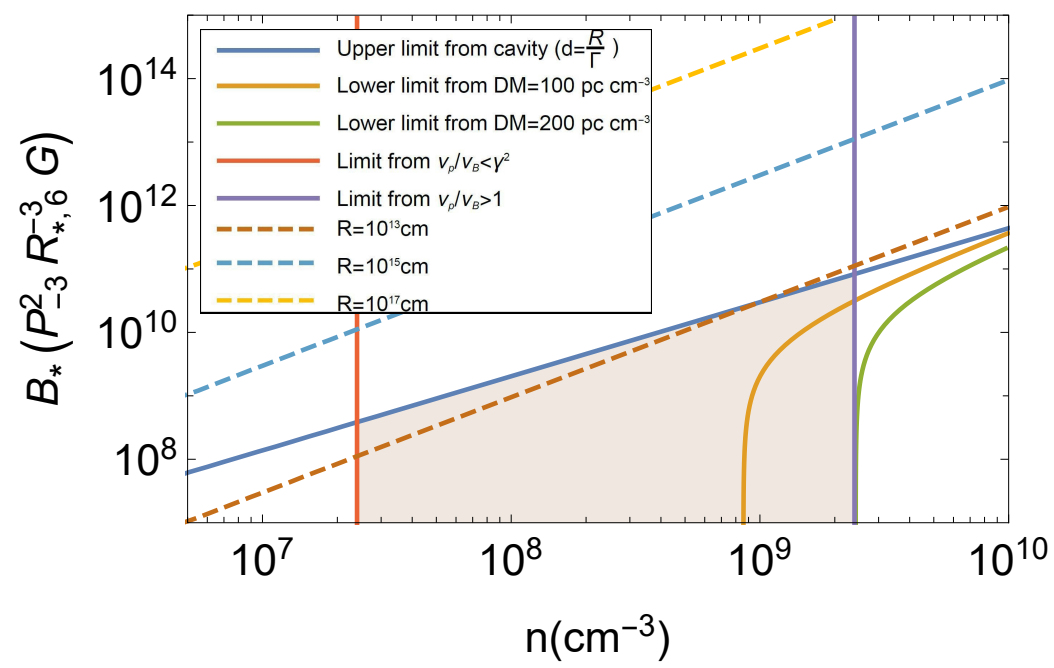

Figure 1: Parameter space (shaded region) for the synchrotron maser with $v_{p} / v_{B}>1, \gamma=10, E=10^{40}$ erg and $\eta=10^{-3}$. Solid lines show limits while the dashed lines show lines of constant radius. Values of $B_{*} \lesssim 10^{10} \mathrm{G}, n \sim 10^{8} \mathrm{~cm}^{-3}$ and $R \sim 10^{13} \mathrm{~cm}$ are preferred. For larger DM values the lower limit will decrease. Increasing the value of $\gamma$ results in less restrictive DM constraints, lower allowed number densities and higher allowed neutron star surface magnetic field values.

We find that a neutron star with a surface magnetic field of $B_{*} \lesssim 10^{10}-10^{11} \mathrm{G}$ is required for emission at the appropriate frequency and energy, increasing to $B_{*} \lesssim 10^{12} \mathrm{G}$ only in the extremely

\footnotetext{
${ }^{2}$ http://www.atnf.csiro.au/research/pulsar/psrcat
} 
relativistic $\gamma=10^{6}$ case. Ruling out pulsars with magnetic fields greater than $10^{11} \mathrm{G}$ leaves approximately $14.5 \%$ of the total population (36). For FRBs with lower energy and greater efficiency, the upper limit on the magnetic field can be significantly lower at $\sim 10^{9.5} \mathrm{G}$. Less than $10 \%$ of pulsars have magnetic fields lower than this value. These upper limits on $B_{*}$ are thus very strong constraints as they rule out the majority of pulsars as being possible hosts for the synchrotron maser in the context of FRBs. The $\sim 15 \%$ of the known pulsar population that meet the criteria are therefore candidates to be FRB progenitors. Therefore, the FRB rate should be a similar fraction of the neutron star formation rate. The neutron star formation rate is approximated by the core-collapse supernova rate which is approximately $\mathscr{R}_{S N} \sim(1.42 \pm 0.3) \times 10^{5} \mathrm{Gpc}^{-3} \mathrm{yr}^{-1}$ (40), while the rate of FRBs is approximately $\mathscr{R}_{F R B} \sim 0.98_{-0.89}^{+1.15} \times 10^{4} \mathrm{Gpc}^{-3} \mathrm{yr}^{-1}$ (2). The ratio of the two rates is $\mathscr{R}_{F R B} / \mathscr{R}_{S N} \sim 0.07$. This value is similar to the fraction of pulsars with surface magnetic fields of less than $10^{10} \mathrm{G}$, which is $\sim 0.1$.

The limits obtained from $D M_{\text {shell }}$ also constrain our results significantly. They have a particularly marked effect in the cases with larger numbers of particles in the masing region. For the higher energy bursts the DM limits severely constrain the cases with lower Lorentz factors, while for the lower energy bursts they are only relevant for $\Gamma=2$. The lower limit on $n_{e}$ depends on the case under consideration. Bursts with higher energies and Lorentz factors have lower allowed number densities. The lowest density of $\sim 1 \mathrm{~cm}^{-3}$ was achieved for $\gamma=10^{6}$.

\section{Discussion}

Emission from the synchrotron maser can be circularly, elliptically or approximately linearly polarised, depending on the electron distribution function and plasma parameters $(24 ; 26)$. Similarly, both circular (e.g. 41) and linear polarisation (e.g. 42) has been measured in FRB observations. However, the heterogeneous nature of FRB polarisation measurements to date makes it difficult to draw useful constraints from the data.

The density constraints obtained from the maser can be compared to the densities found in the vicinity of neutron stars. In the case of pulsar wind nebulae, densities of $n \sim 10^{-6} \mathrm{~cm}^{-3}$ and magnetic fields of $B_{M} \sim 10^{-2}-10^{-1} \mathrm{G}$ are expected $(15 ; 39 ; 38 ; 43)$. Neither of these values lie within the allowed parameter space for the synchrotron maser, ruling out this scenario.

In order to account for the larger density values required by our constraints, we are lead to suggest a scenario where weakly magnetised neutron stars undergo an accretion induced explosion (44). The material expelled by this explosion can then form a shell of width $\sim R / \Gamma$ in which a population inversion is formed, and as a result masing takes place. Accreting neutron stars in low mass X-Ray binaries (LMXBs) have typical wind densities of $10^{13}-10^{15} \mathrm{~cm}^{-3}$ at radii of approximately $10^{10} \mathrm{~cm}(45)$. While these density values are too high for the maser, our scenario considers the masing emission to occur at larger distances of $R \sim 10^{13} \mathrm{~cm}$. As, at constant velocity, $n \propto r^{-2}$, the particles from the accretion induced explosion could plausibly provide suitable number densities for the maser at these distances. Pulsars in binary systems with $B_{*}<10^{11} \mathrm{G}$ have typical periods of $\sim$ few ms and make up $\sim 0.09$ of the total population (36), comparable to the ratio of the FRB and neutron star formation rates. As a result, this scenario would require a significant fraction of low magnetic field neutron stars in binaries to undergo such an event due to the similarities 
between the FRB rate and the neutron star formation rate. For further detail about the model, please refer to (46).

\section{References}

[1] E. Petroff, E. D. Barr, A. Jameson, E. F. Keane, M. Bailes, M. Kramer et al., FRBCAT: The Fast Radio Burst Catalogue, Publ. Astron. Soc. Aust. 33 (2016) e045.

[2] D. J. Champion, E. Petroff, M. Kramer, M. J. Keith, M. Bailes, E. D. Barr et al., Five new fast radio bursts from the HTRU high-latitude survey at Parkes: first evidence for two-component bursts, MNRAS 460 (2016) L30.

[3] L. G. Spitler, P. Scholz, J. W. T. Hessels, S. Bogdanov, A. Brazier, F. Camilo et al., A repeating fast radio burst, Nature 531 (2016) 202.

[4] S. P. Tendulkar, C. G. Bassa, J. M. Cordes, G. C. Bower, C. J. Law, S. Chatterjee et al., The Host Galaxy and Redshift of the Repeating Fast Radio Burst FRB 121102, ApJL 834 (2017) L7.

[5] B. Marcote, Z. Paragi, J. W. T. Hessels, A. Keimpema, H. J. van Langevelde, Y. Huang et al., The Repeating Fast Radio Burst FRB 121102 as Seen on Milliarcsecond Angular Scales, ApJL 834 (2017) L8.

[6] S. Chatterjee, C. J. Law, R. S. Wharton, S. Burke-Spolaor, J. W. T. Hessels, G. C. Bower et al., A direct localization of a fast radio burst and its host, Nature 541 (2017) 58.

[7] K. W. Bannister, A. T. Deller, C. Phillips, J. P. Macquart, J. X. Prochaska, N. Tejos et al., A single fast radio burst localized to a massive galaxy at cosmological distance, Science 365 (2019) 565 [1906.11476].

[8] V. Ravi, M. Catha, L. D’Addario, S. G. Djorgovski, G. Hallinan, R. Hobbs et al., A fast radio burst localised to a massive galaxy, arXiv e-prints (2019) arXiv:1907.01542 [1907.01542].

[9] J. X. Prochaska, J.-P. Macquart, M. McQuinn, S. Simha, R. M. Shannon, C. K. Day et al., The low density and magnetization of a massive galaxy halo exposed by a fast radio burst, Science 366 (2019) 231.

[10] A. L. Piro, Magnetic Interactions in Coalescing Neutron Star Binaries, ApJ 755 (2012) 80.

[11] K. Kashiyama, K. Ioka and P. Mészáros, Cosmological Fast Radio Bursts from Binary White Dwarf Mergers, ApJL 776 (2013) L39.

[12] C. M. F. Mingarelli, J. Levin and T. J. W. Lazio, Fast Radio Bursts and Radio Transients from Black Hole Batteries, ApJL 814 (2015) L20.

[13] E. F. Keane, B. W. Stappers, M. Kramer and A. G. Lyne, On the origin of a highly dispersed coherent radio burst, MNRAS 425 (2012) L71. 
[14] J. M. Cordes and I. Wasserman, Supergiant pulses from extragalactic neutron stars, MNRAS 457 (2016) 232.

[15] Y. Lyubarsky, A model for fast extragalactic radio bursts, MNRAS 442 (2014) L9.

[16] A. M. Beloborodov, A Flaring Magnetar in FRB 121102?, ApJL 843 (2017) L26.

[17] J. I. Katz, Fast radio bursts - A brief review: Some questions, fewer answers, Mod. Phys. Lett A 31 (2016) 1630013.

[18] P. Kumar, W. Lu and M. Bhattacharya, Fast radio burst source properties and curvature radiation model, MNRAS 468 (2017) 2726.

[19] G. Ghisellini and N. Locatelli, Coherent curvature radiation and Fast Radio Bursts, .

[20] E. Waxman, On the Origin of Fast Radio Bursts (FRBs), ApJ 842 (2017) 34.

[21] G. Ghisellini, Synchrotron masers and fast radio bursts, MNRAS 465 (2017) L30.

[22] G. E. Romero, M. V. del Valle and F. L. Vieyro, Mechanism for fast radio bursts, PRD 93 (2016) 023001.

[23] C. S. Wu, Kinetic cyclotron and synchrotron maser instabilities - Radio emission processes by direct amplification of radiation, Space Sci. Rev. 41 (1985) 215.

[24] R. A. Treumann, The electron-cyclotron maser for astrophysical application, Astron. Astrophys. Rev. 13 (2006) 229.

[25] G. B. Rybicki and A. P. Lightman, Radiative processes in astrophysics. 1979.

[26] A. Sagiv and E. Waxman, Collective Processes in Relativistic Plasma and Their Implications for Gamma-Ray Burst Afterglows, ApJ 574 (2002) 861.

[27] R. McCray, Possibility of Maser Action in Cosmic Radio Sources, Science 154 (1966) 1320.

[28] V. V. Zheleznyakov, A Coherent Synchrotron Mechanism for Radio Emission from Cosmic Sources., Sov. Ast. 11 (1967) 33.

[29] R. D. Blandford and C. F. McKee, Fluid dynamics of relativistic blast waves, Physics of Fluids 19 (1976) 1130.

[30] C. J. Law, M. W. Abruzzo, C. G. Bassa, G. C. Bower, S. Burke-Spolaor, B. J. Butler et al., A Multi-telescope Campaign on FRB 121102: Implications for the FRB Population, ApJ 850 (2017) 76.

[31] Y. A. Gallant, M. Hoshino, A. B. Langdon, J. Arons and C. E. Max, Relativistic, perpendicular shocks in electron-positron plasmas, ApJ 391 (1992) 73.

[32] L. Sironi and A. Spitkovsky, Particle Acceleration in Relativistic Magnetized Collisionless Pair Shocks: Dependence of Shock Acceleration on Magnetic Obliquity, ApJ 698 (2009) 1523. 
[33] W. Lu and P. Kumar, On the radiation mechanism of repeating fast radio bursts, MNRAS 477 (2018) 2470 [1710.10270].

[34] G. Benford and H. Lesch, Coherent emission and the escape of high brightness temperature radiation in active galactic nuclei, MNRAS 301 (1998) 414.

[35] R. V. Shcherbakov, Propagation Effects in Magnetized Transrelativistic Plasmas, ApJ $6 \mathbf{8 8}$ (2008) 695 .

[36] R. N. Manchester, G. B. Hobbs, A. Teoh and M. Hobbs, The Australia Telescope National Facility Pulsar Catalogue, AJ 129 (2005) 1993.

[37] P. Goldreich and W. H. Julian, Pulsar Electrodynamics, ApJ 157 (1969) 869.

[38] B. M. Gaensler and P. O. Slane, The Evolution and Structure of Pulsar Wind Nebulae, Annu. Rev. Astron. Astrophys. 44 (2006) 17.

[39] J. G. Kirk, Y. Lyubarsky and J. Petri, The Theory of Pulsar Winds and Nebulae, in Astrophysics and Space Science Library, W. Becker, ed., vol. 357 of Astrophysics and Space Science Library, p. 421, 2009, DOI.

[40] G. Bazin, N. Palanque-Delabrouille, J. Rich, V. Ruhlmann-Kleider, E. Aubourg, L. Le Guillou et al., The core-collapse rate from the Supernova Legacy Survey, A\&A 499 (2009) 653.

[41] K. Masui, H.-H. Lin, J. Sievers, C. J. Anderson, T.-C. Chang, X. Chen et al., Dense magnetized plasma associated with a fast radio burst, Nature 528 (2015) 523.

[42] D. Michilli, A. Seymour, J. W. T. Hessels, L. G. Spitler, V. Gajjar, A. M. Archibald et al., An extreme magneto-ionic environment associated with the fast radio burst source FRB 121102, Nature 553 (2018) 182.

[43] B. Olmi, L. Del Zanna, E. Amato, R. Bandiera and N. Bucciantini, On the magnetohydrodynamic modelling of the Crab nebula radio emission, MNRAS 438 (2014) 1518.

[44] J. I. Katz, H. A. Toole and S. H. Unruh, Yet another model of soft gamma repeaters, ApJ 437 (1994) 727 [astro-ph/9404039].

[45] M. Díaz Trigo and L. Boirin, Accretion disc atmospheres and winds in low-mass X-ray binaries, Astronomische Nachrichten 337 (2016) 368.

[46] K. Long and A. Pe'er, Synchrotron Maser from Weakly Magnetized Neutron Stars as the Emission Mechanism of Fast Radio Bursts, ApJL 864 (2018) L12 [1806 . 0270 0]. 